\title{
PATRONES QUIMICOS Y ORIGEN DEL DEPOSITO DE ORO DE LA LIBERTAD, CHONTALES, NICARAGUA
}

\author{
Mauricio Darce', Beatriz Levi² \& Olav Nyström ${ }^{3}$ \\ ") Empresa Minera de Occidente S.A., RP-01 Managua, Nicaragua \\ ${ }^{2)}$ Department of Geology and Geochemistry, Stockholm University, S-10691 \\ Stockholm, Sweden
${ }^{3)}$ Swedish Museum of Natural History, S-104505 Stockholm, Sweden
}

(Recibido 5/5/93, Aceptado 27/6/94)

\begin{abstract}
The La Liberad region is situated in the central part of Nicaragua, within a broad discontinuous belt of Tertiary volcanic rocks that contains several epithermal gold and silver deposits and extends from Guatemala (and Mexico) to Costa Rica.

A chemical comparision between altered and unaltered basic lavas of this area shows that chemical changes associated with the geothermal field type of alteration centered at the mining district reach more than $5 \mathrm{~km}$ away from it. Water content, $\mathrm{CO}_{2}, \mathrm{~K}$ and $\mathrm{S}$ have beeen added, titanium seems to have been inmobile and $\mathrm{Cl}$ partily lost from the fossil geothermal system. Gold, originally concentrated in the glass of basic lavas, was leached during zeolite facies conditions and precipitated with silica in fractures, forming veins on the center of the geothermal field. An estimate shows that the amount of Au released during the alteration was sufficient to give origin to La Libertad deposit.

RESUMEN: La región de La Libertad en la parte central de Nicaragua está constituida por rocas volcánicas Terciarias de composición básica y alteradas en la facies de ceolitas. La alteración define patrones específicos de tipo mineralógico y químico a diferentes escalas. Diagénesis de sepultamiento de carácter regional (Zona I) grada hacia zonas alteradas que se han formado en un campo geotérmico (Zonas II-IV), centradas en un enjambre de vetas de cuarzo que contienen oro en el área del distrito minero de La Libertad. La zona V coincide con una capa caoliníticailítica supergénica que cubre a la mayoría del distrito. Las aureolas de las vetas presentan un patrón de alteración de carácter local.

La hidratación y la oxidación durante la diagénesis de sepultamiento se caracterizó por una relativamente fácil movilidad de elementos $\mathrm{K}, \mathrm{Ni}, \mathrm{Mg}$ y Cl . Agua, $\mathrm{CO}_{2}, \mathrm{~K}$ (liberados durante la diagénesis regional) y $\mathrm{S}$ han sido adicionados; $\mathrm{Ni}, \mathrm{Mg} \mathrm{y} \mathrm{Cl}$ fueron parcialmente perdidos del sistema geotérmico, el cual se extendió más de $5 \mathrm{~km}$ del presente distrito minero. Algunos elementos $(\mathrm{Si}, \mathrm{Ti}, \mathrm{Al}, \mathrm{Fe}, \mathrm{Mn}$ y $\mathrm{Na}$ ) varían muy poco entre las diferentes zonas de alteración del sistema fósil. El Ca fue fuertemente sustraído en las aureolas y la capa caolinítica; el $\mathrm{Zr}$ y el Y muestran evidencias de movilidad en las aureolas.

El oro fué sustraído de las muestras de afloramientos mientras que el Cu varía muy poco. Este comportamiento contrastante se debe a la lixiviación del oro y la formación de sulfuros de Cu remanentes en las rocas alteradas. La fuerte lixiviación del oro tomó lugar en la zona II, en donde una de las mayores reacciones fué el reemplazamiento del vidrio volcánico por esmectita. El oro y el $\mathrm{Cu}$ fueron probablemente concentrados en la mezcla silicática residual (vidrio) de las lavas básicas. El oro lixiviado fué precipitado junto con el sílice en las fracturas, formándose de esta manera las vetas de cuarzo auriferas. Una estimación preliminar muestra que la cantidad del oro liberado durante la alteración fué suficiente para formar el depósito de La Libertad. Los patrones químicos-mineralógicos a diferentes escalas sugieren que la alteración en el sistema geotérmico fósil y en las aureolas debajo de la capa caolinítica fueron expresiones simultaneas del mismo proceso.
\end{abstract}

\section{INTRODUCCION}

Depósitos epitermales de metales preciosos ocurren principalmente en rocas volcánicas altera- das y particularmente son comunes en las fajas volcánicas Cenozoicas de la Región Circum-Pacífica. Ha sido sugerido por largo tiempo que los sistemas geotérmicos activos son los análogos 
modernos de los depósitos epitermales (Lindgren, 1928, White, 1955, 1981), de que el oro y la plata de estos depósitos podrían proceder de la roca volcánica de caja y que se han lixiviado durante los procesos de alteración. Esta opinión es soportada por evidencias indirectas, tales como estudios de isótopos de $\mathrm{Pb}$ y otros (Field \& Fifarek, 1985; Hayba et al., 1985). Boyle (1979, p.395) menciona que las rocas volcánicas circundantes a un sistema hidrotermal pueden ser la fuente del oro en los depósitos epitermales. Sin embargo, solo se conoce de una investigación (Dostal \& Dupuy, 1987) que muestra una relación entre la alteración de la roca volcánica y la lixiviación de oro, pero la alteración descrita es de un carácter regional (rocas Proterozoicas alteradas en la facies de Prehenita-Pumpelita) y no se mencionan depósitos epitermales.

En Nicaragua, las vetas epitermales de cuarzo aurífero ocurren en diversos enjambres, los cuales son objeto de explotación industrial. El propósito de esta investigación es el de describir y discutir los cambios químicos relacionados con la formación de uno de los más representativos enjambres de vetas auríferas como lo es el depósito de oro de La Libertad, el cual está ubicado en la parte central de Nicaragua. Se ha puesto especial énfasis en el comportamiento geoquímico del oro y del cobre y se demuestra de que durante procesos de alteración hidrotermal el oro fue lixiviado de la roca volcánica circundante, en un área mucho mayor de la del distrito minero própiamente. También se aportan evidencias de que gran parte del oro originalmente estaba alojado en el vidrio de la roca volcánica circundante. La historia geológica del área y los cambios mineralógicos que ocurrieron durante la alteración fueron descritos por Darce (1989).

El distrito minero de La Libertad está situado dentro de una amplia faja discontínua de rocas volcánicas Terciarias, la cual se extiende desde Guatemala (y Mexico) hasta Costa Rica (Fig.1). Esta faja contiene varios depósitos epitermales de oro y plata (Dengo \& Levy, 1970; Sillitoe, 1977).

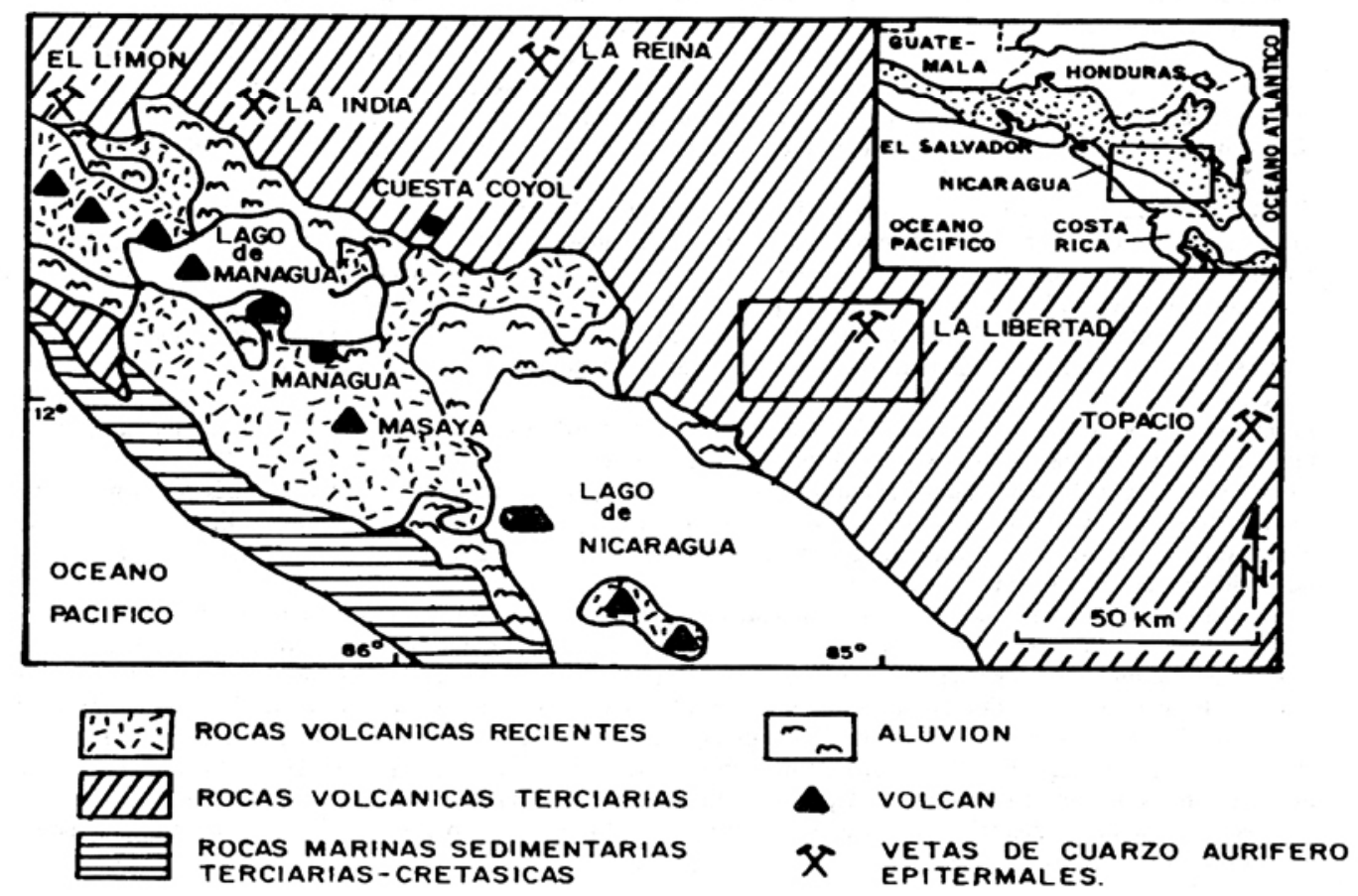

Fig. 1: Mapa geológico simplificado de la parte central de Nicaragua (modificado del Mapa Geológico preliminar $1: 1.000 .000$, Nicaragua 1993). Los depósitos de cenizas y lahares Pliocenicos Pleistocenicos del Grupo de la Sierras están incluidos con la rocas volcánicas recientes. Los productos volcánicos recientes, depósitos aluviales y los dos grandes lagos están ocupando la estructura volcano-tectónica de la Depresión de Nicaragua. El área de La Libertad (Fig.2) está ubicado en un marco. Las secuencias volcánicas Terciarias (Dengo, 1965) y los depósitos epitermales de metales preciosos asociados en otras partes de Centroamerica (Dengo \& Levi, 1970; Levi 1970; Sillitoe, 1977) están mostrados en el cuadro inserto. ( 1 = Monte Cristo, 2 = Rosario, $3=$ El Chorreadero, $4=$ Bonanza y $5=$ Boston). 
En la región de La Libertad (Fig.2) el oro ocurre en un enjambre de vetas de cuarzo alojados en una pila de rocas volcánicas predominantemente de carácter basáltico (parte inferior del Grupo
Coyol), la cual tiene un espesor aflorante de aproximadamente $400 \mathrm{~m}$; el depósito es del tipo adularia sericítico (Darce, 1989).
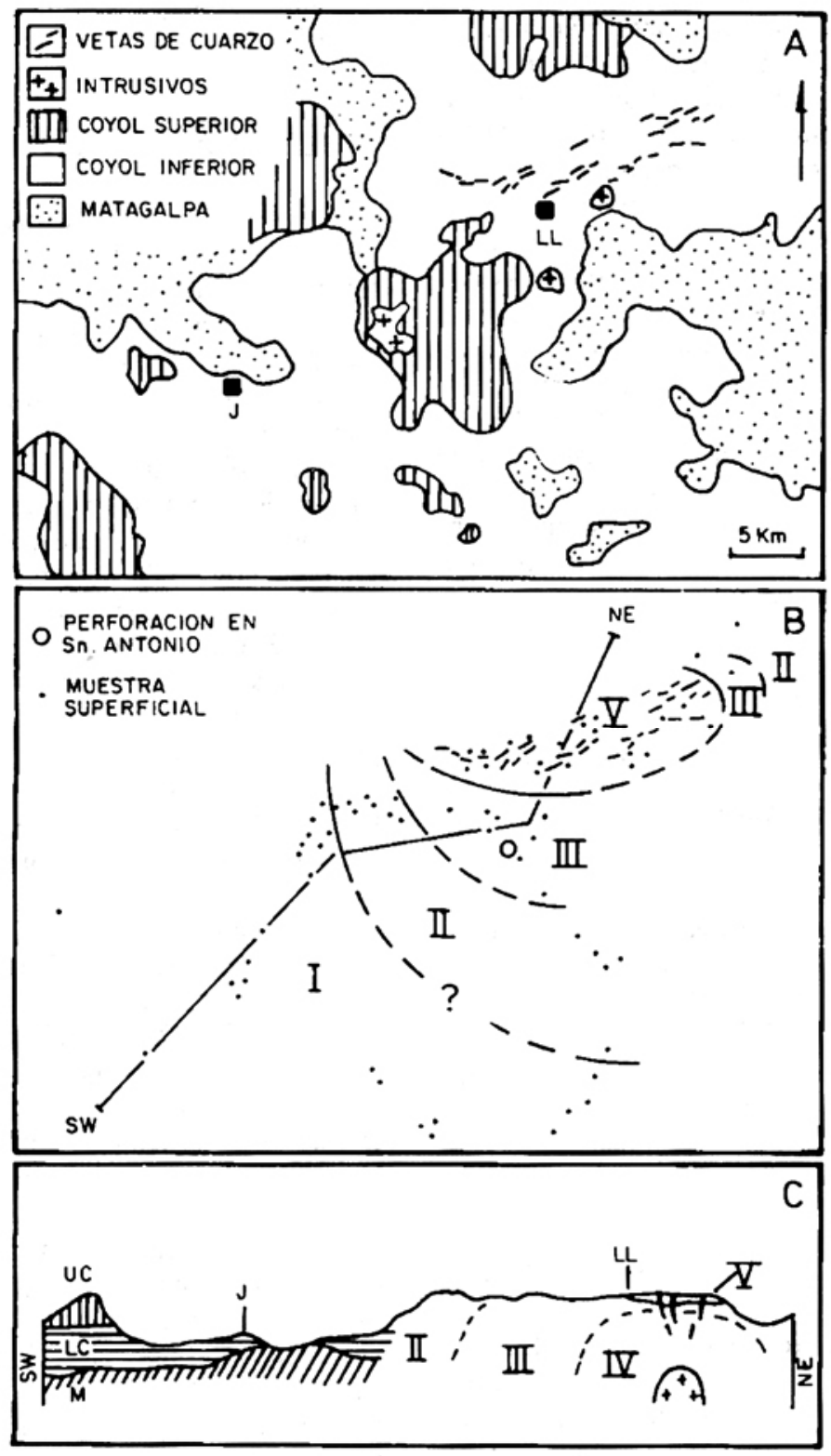

Fig. 2: La región de La Libertad en la parte central de Nicaragua: $A=$ mapa geológico ( $\mathrm{J}=$ Guigalpa, $\mathrm{LL}=\mathrm{La}$ Libertad; simplificado de Darce 1978), B = Zonas de alteración basadas en asociaciones de minerales secundarios en la Unidad Coyol Inferior (Darce, 1989; los limites de la zona II hacia el SW de La Libertad son muy inseguros debido a la desigualdad de las muestras; la zona V es una capa caolinítica-ilítica, la cual está cubriendo la mayoría del distrito minero), y C = perfil esquemático; la yacencia en la pila volcánica es mostrado unicamente para la parte exterior del sistema geotérmico fosil (= zona I; UC = Unida de Coyol Superior, LC = Unidad del Coyol Inferior y M = Grupo de Matagalpa). Las vetas auríferas de cuarzo y sus aureolas están representadas en bordes negros. La ubicación de las perforaciones y el túnel de acceso a Santa Elena esta mostrado en Darce (1989, Fig.4). 
Las rocas volcánicas contienen minerales secundarios formados por diferentes procesos de alteración que varían desde diagénesis a escala regional a un tipo de alteración geotérmico centrado en La Libertad; también se dan alteraciones locales en las aureolas de las vetas de cuarzo. Cambios sistemáticos en las alteraciones de minerales secundarios en las rocas de la unidad Coyol Inferior y las vetas han sido reconocidas por Darce (1989) y subdivididos en cinco zonas especificas de alteración (Fig.2B-C; tabla 1). Las rocas en la Zona I (diagénesis de sepultamiento regional; subfacies de mordenita) están esencialmente no alteradas con excepción de las partes originalmente permeables. La extensión y el grado de la alteración se volvió sucesivamente mayor hacia el sistema geotérmico fósil, desde la Zona II con subfacies de heulandita hasta la Zona IV con subfacies de transición entre laumontita y wairakita. La Zona V es una capa supergénica de 30-40 $\mathrm{m}$ de espesor constituida principalmente de caolinita-ilita y que está cubriendo la mayor parte del distrito.

Tabla 1

\begin{tabular}{ll} 
& $\begin{array}{c}\text { Los minerales secundarios más importantes en las rocas } \\
\text { volcánicas básicas del Grupo Coyol Inferior, región } \\
\text { de La Libertad }\end{array}$ \\
\hline Zona & $\begin{array}{l}\text { Minerales secundarios } \\
\text { I }\end{array}$ \\
II & $\begin{array}{l}\text { Esmectita, heulandita, cristobalita, tridimita y } \\
\text { mordenita }\end{array}$ \\
III & $\begin{array}{l}\text { Esmectita, cuarzo y heulandita } \\
\text { Cuarzo, clorita expandible (esmectita), calcita y } \\
\text { laumontita }\end{array}$ \\
IV & $\begin{array}{l}\text { Clorita, cuarzo, calcita, laumontita (o wairakita) } \\
\pm \text { epidota, ilita, caolinita }\end{array}$ \\
V & $\begin{array}{l}\text { Cuarzo, caolinita, ilita y pirita } \\
\text { V }\end{array}$
\end{tabular}

Las vetas de cuarzo ocurren en un área de aproximadamente $20 \times 5 \mathrm{~km}$ (Fig.2), son subverticales con un ancho entre 0,5 a 20 m cerca de la superficie y más estrechas en la profundidad. Consisten de cuarzo lechoso con adularia e ilita. El oro está presente como electrum asociado con pirita y localmente trazas de calcopirita, galena, esfalerita y minerales de plata (Hålenius, 1983). Las vetas están rodeadas de aureolas compuestas de cuarzo, pirita, clorita, adularia e ilita bajo la capa de caolinita-ilita en la superficie. Las reservas minerales (probadas más probables) son de alrededor de $1,3 * 10^{6}$ tonelas con un promedio de 0,15 gr/ton (INMINE, datos no publicados). Esta cifra no toma en cuenta el oro que ha sido removido por la erosión natural, cuyo nivel se estima entre 100 a $370 \mathrm{~m}$ en la mayoría de la vetas que afloran en la superficie (Darce, 1989).

\section{METODOS ANALITICOS Y MUESTREO}

Los elementos mayores y algunos menores han sido determinados en 88 muestras provenientes de la Zona I de la región de La Libertad y en 6 muestras afuera de la Zona I. Muestras con $\mathrm{H}_{2} \mathrm{O}<$ $1 \%$ del peso total y $\mathrm{CO}_{2}<0,2 \%$ se consideran como no alteradas; las otras fueron clasificadas como alteradas.

Los elementos mayores y $\mathrm{Ba}, \mathrm{Cu}, \mathrm{Ni}, \mathrm{Sr}$ y $\mathrm{V}$ fueron determinados por espectrometría de emisión óptica (ICP) en Lulea, Suecia (C. Pontér, Servicio Geológico de Suecia, SGAB). Algunos análisis fueron repetidos en la Universidad de Estocolmo, Suecia (B. Boström y M. Darce) y dieron resultados muy similares. Para el presente trabajo solo se utilizaron los datos del SGAB. Se aplicó el procedimiento analítico de Burman et al. (1977, 1978), con la modificación de que la digestión del metaborato de litio fue usado solamente para los elementos trazas, lo cual mejoró la precisión del método.

$\mathrm{El}$ agua $\left(\mathrm{H}_{2} \mathrm{O}^{+}\right), \mathrm{CO}_{2}$ y $\mathrm{FeO}$ en todas las muestras y el S y Cl en 45 muestras fueron obtenidos por métodos químicos húmedos en el "Centre de Recherches Pétrographiques et Geochimiques CRPG, Nancy, Francia). El itrium fue determinado en 62 muestras y el $\mathrm{Zr}$ en 58 por espectrometría de emisión óptica (combinado con intercambio de separación iónica por Y) en el CRPG (Govindaráju \& Mevelle, 1987). El oro fue determinado en 50 muestras por análisis de activación nuclear radiogeoquímica y ensayo por fuego por separación de Au después de la irradiación en "Nuclear Activation Services Ltd.", Hamilton, Ontario, Canadá. El valor del Au para cada muestra representa el promedio de dos análisis independientes. Numerosas repeticiones indican que la desviación estandar de 1 gramo de muestra con una concentración de Au por debajo de 1 ppb es de alrededor de 0,1 ppb. Valores de densidad seca de 16 muestras analizadas fueron medidas por $\mathrm{K}$. Ubieta en el Departamento de Geofísica Aplicada de la Universidad de Lulea, Suecia. 
Tabla 2

Composición química promedio y desviaciones estandares de las muestras de afloramientos de lavas básicas (y vetas de cuarzo) del Grupo Coyol Inferior en las diferentes zonas de alteración de la región de La Libertad. Las muestras del valor de fondo son no alteradas; todas las otras muestras de rocas son alteradas. La capa caolinítica (zona IV) incluye muestras de aureolas de vetas. $\mathrm{Fe} 0$ * es hierro total; $\mathrm{FM}=(\mathrm{Fe} 0 *+\mathrm{MnO}) /(\mathrm{Fe} 0 *+\mathrm{Mn} 0+\mathrm{Mg} 0) ; 0 \mathrm{X}=\mathrm{Fe}_{2} 0_{3} / \mathrm{Fe} 0 ; \mathrm{n}=$ número de muestras analizadas. Los elementos mayores, $\mathrm{H}_{2} \mathrm{O}$ y CO $\mathrm{CO}_{2}$ están dados en \% de peso, Au en ppb y los otros elementos en ppm .

\begin{tabular}{|c|c|c|c|c|c|c|}
\hline \multirow{2}{*}{$\begin{array}{l}\mathrm{K} \\
\mathrm{SiO}_{2}\end{array}$} & \multirow{2}{*}{$\begin{array}{c}\begin{array}{c}\text { Zona I } \\
\text { Valor fondo }\end{array} \\
50,2 \pm 0,6\end{array}$} & \multirow{2}{*}{$\begin{array}{c}\text { Zona II } \\
56,0 \pm 5,0\end{array}$} & \multirow{2}{*}{$\begin{array}{c}\text { Zona III } \\
58,1 \pm 6,8\end{array}$} & \multirow{2}{*}{$\begin{array}{c}\text { Zona IV } \\
53,7 \pm 10,3\end{array}$} & \multicolumn{2}{|c|}{$\begin{array}{c}\text { Zona V } \\
\text { Super Vetas }\end{array}$} \\
\hline & & & & & $57,5 \pm 9,7$ & $91,7 \pm 5,5$ \\
\hline $\mathrm{TiO}_{2}^{2}$ & $0,87 \pm 0,09$ & $1,05 \pm 0,15$ & $0,93 \pm 0,17$ & $0,82 \pm 0,11$ & $1,11 \pm 0,24$ & $0,16 \pm 0,16$ \\
\hline $\mathrm{Al}_{2} \mathrm{O}_{3}$ & $18,7 \pm 1,5$ & $16,4 \pm 1,3$ & $16,0 \pm 1,5$ & $18,0 \pm 2,9$ & $20,9 \pm 5,5$ & $3,5 \pm 2,9$ \\
\hline $\mathrm{FeO}$ & $9,3 \pm 0,45$ & $8,74 \pm 0,94$ & $8,02 \pm 1,8$ & $7,75 \pm 2,43$ & $8,48 \pm 4,83$ & $1,84 \pm 1,27$ \\
\hline $\mathrm{MnO}$ & $0,18 \pm 0,06$ & $0,18 \pm 0,05$ & $0,20 \pm 0,17$ & $0,15 \pm 0,04$ & $0,09 \pm 0,17$ & $0,14 \pm 0,22$ \\
\hline $\mathrm{MgO}$ & $5,86 \pm 1,35$ & $3,95 \pm 2,71$ & $2,74 \pm 1,69$ & $3,55 \pm 1,91$ & $0,54 \pm 0,38$ & $0,10 \pm 0,11$ \\
\hline $\mathrm{CaO}$ & $10,28 \pm 0,49$ & $6,78 \pm 2,71$ & $6,09 \pm 4,01$ & $6,07 \pm 3,22$ & $0,08 \pm 0,12$ & $0,06 \pm 0,01$ \\
\hline $\mathrm{Na}_{2} \mathrm{O}$ & $2,54 \pm 0,22$ & $3,16 \pm 0,48$ & $2,47 \pm 0,87$ & $2,67 \pm 0,75$ & $0,19 \pm 0,55$ & $0,05 \pm 0,02$ \\
\hline $\mathrm{K}_{2} \mathrm{O}$ & $0,38 \pm 0,18$ & $1,32 \pm 0,95$ & $1,63 \pm 1,42$ & $1,47 \pm 1,82$ & $2,53 \pm 1,90$ & $0,57 \pm 0,65$ \\
\hline $\mathrm{P}_{2} \mathrm{O}_{5}$ & $0,16 \pm 0,07$ & $0,32 \pm 0,17$ & $0,20 \pm 0,04$ & $0,18 \pm 0,10$ & $0,11 \pm 0,07$ & $0,06 \pm 0,06$ \\
\hline $\mathrm{H}_{2} \mathrm{O}^{+}$ & $0,85 \pm 0,14$ & $1,83 \pm 0,85$ & $3,82 \pm 2,18$ & $3,43 \pm 1,49$ & $6,58 \pm 2,94$ & $1,22 \pm 0,56$ \\
\hline $\mathrm{CO}_{2}$ & $0,11 \pm 0,04$ & $0,15 \pm 0,08$ & $0,92 \pm 1,06$ & $1,15 \pm 1,02$ & $0,13 \pm 0,06$ & $0,05 \pm 0,01$ \\
\hline $\mathrm{s}$ & $38 \pm 5$ & $43 \pm 6$ & $50 \pm 14$ & $70 \pm 28$ & $200 \pm 190$ & $55 \pm 6$ \\
\hline $\mathrm{Cl}$ & $68 \pm 25$ & $77 \pm 25$ & $48 \pm 26$ & $32 \pm 11$ & $48 \pm 41$ & $15 \pm 0$ \\
\hline $\mathrm{Ba}$ & $270 \pm 55$ & $530 \pm 280$ & $580 \pm 420$ & $700 \pm 480$ & $320 \pm 260$ & $230 \pm 300$ \\
\hline $\mathrm{Sr}$ & $460 \pm 31$ & $410 \pm 100$ & $190 \pm 120$ & $380 \pm 120$ & $40 \pm 46$ & $16 \pm 8$ \\
\hline $\mathrm{Ni}$ & $61 \pm 36$ & $38 \pm 44$ & $15 \pm 15$ & $31 \pm 24$ & $10 \pm 6$ & $9 \pm 6$ \\
\hline $\mathrm{V}$ & $280 \pm 23$ & $220 \pm 100$ & $200 \pm 92$ & $170 \pm 120$ & $280 \pm 120$ & $27 \pm 21$ \\
\hline $\mathrm{Cu}$ & $106 \pm 17$ & $81 \pm 36$ & $107 \pm 82$ & $80 \pm 26$ & $97 \pm 92$ & $44 \pm 25$ \\
\hline $\mathrm{Au}$ & $3,6 \pm 0,7$ & $0,9 \pm 0,7$ & $1,8 \pm 1,0$ & $1,9 \pm 1,0$ & $16 \pm 21$ & $2000 \pm 1600$ \\
\hline $\mathrm{Zr}$ & $60 \pm 17$ & $160 \pm 100$ & $120 \pm 63$ & $130 \pm 120$ & $95 \pm 67$ & $64 \pm 50$ \\
\hline Y & $20 \pm 3$ & $38 \pm 19$ & $31 \pm 6$ & $32 \pm 28$ & $29 \pm 17$ & $11 \pm 15$ \\
\hline FM & $0,62 \pm 0,06$ & $0,69 \pm 0,12$ & $0,77 \pm 0,10$ & $0,69 \pm 0,07$ & $0,93 \pm 0,05$ & $0,95 \pm 0,06$ \\
\hline $\mathrm{OX}$ & $0,99 \pm 0,36$ & $4,4 \pm 23$ & $13 \pm 14$ & $1,7 \pm 1,7$ & $67 \pm 105$ & $31 \pm 84$ \\
\hline $\mathrm{n}$ & 6 & 6 & 5 & 3 & 13 & 7 \\
\hline
\end{tabular}

A fin de estudiar las ganancias y pérdidas geoquímicas que ocurrieron durante los procesos de alteración es necesario conocer los contenidos de varios elementos en las rocas no alteradas, por ejemplo, los valores de fondo. El valor de fondo corresponde al de la composición promedio de las muestras de basaltos no alterados de la unidad Coyol Inferior de la Zona I. Las muestras provienen de las partes masivas de flujos lávicos que virtualmente no fueron afectas por la alteración y contienen únicamente esmectita proveniente del olivino. La composición de los basaltos de Coyol Inferior fue escogida como valor de fondo debido a que este es el tipo de roca predominante en los alrededores del depósito de La Libertad. Minerales seudomórficos provenientes de la alteración del olivino son muy abundantes en las lavas de la zona II y IV, indicando su carácter básico. Textural y mineralógicamente estas rocas se parecen a los basaltos tomados como valores de fondo ya que contienen relictos de fenocristales similares a los piroxenos y plagioclasas cálcicas de la Zona I.

Composiciones promedios fueron calculados para las rocas básicas alteradas en cada zona del campo geotérmico fósil; lo mismo se hizo para un grupo de muestras de los afloramientos y de otro grupo de núcleos provenientes de las perforaciones. Las muestras de superficie y de sub-superficie fueron tratadas separadamente debido a que como grupos ellos difieren en la extensión de la oxidación y contenidos de algunos elementos. Otra razón para la separación de estos grupos es que el comportamiento geoquímico de las rocas superficiales es de interés para la prospección de mineralizaciones auriferas del tipo de La Libertad. Las muestras de núcleos de perforaciones de la zona IV fueron subdivididos en rocas y aureolas. Las muestras de la zona $V$ fueron subdivididas en dos sub-grupos: Las rocas de la capa caolinítica (incluyendo muestras de aureolas) y las vetas de cuarzo. 
Tabla 3

\begin{tabular}{|c|c|c|c|c|}
\hline & $\begin{array}{c}\text { Zona I } \\
\text { Valor fondo }\end{array}$ & $\begin{array}{c}\text { Zona III } \\
\text { San Antonio }\end{array}$ & Roca (SJ) & Aureola \\
\hline $\mathrm{SiO}_{2}$ & $50,2 \pm 0,6$ & $50,6 \pm 2,7$ & $49,2 \pm 2,5$ & $63,2 \pm 8,4$ \\
\hline $\mathrm{TiO}_{2}^{2}$ & $0,87 \pm 0,09$ & $0,85 \pm 0,10$ & $0,91 \pm 0,16$ & $0,65 \pm 0,14$ \\
\hline $\mathrm{Al}_{2} \mathrm{O}_{3}$ & $18,7 \pm 1,5$ & $16,7 \pm 1,7$ & $18,8 \pm 2,1$ & $16,8 \pm 3,5$ \\
\hline $\mathrm{FeO}$ & $9,30 \pm 0,45$ & $8,70 \pm 0,79$ & $9,19 \pm 1,32$ & $6,33 \pm 2,19$ \\
\hline $\mathrm{MnO}$ & $0,18 \pm 0,06$ & $0,30 \pm 0,09$ & $0,19 \pm 0,04$ & $0,15 \pm 0,07$ \\
\hline $\mathrm{MgO}$ & $5,86 \pm 1,35$ & $6,28 \pm 1,38$ & $3,99 \pm 0,60$ & $2,31 \pm 1,86$ \\
\hline $\mathrm{CaO}$ & $10,28 \pm 0,49$ & $8,53 \pm 2,09$ & $10,40 \pm 2,61$ & $0,73 \pm 0,13$ \\
\hline $\mathrm{Na}_{2} \mathrm{O}$ & $2,54 \pm 0,22$ & $2,75 \pm 0,38$ & $2,07 \pm 0,79$ & $2,37 \pm 2,10$ \\
\hline $\mathrm{K} 2 \mathrm{O}$ & $0,38 \pm 0,18$ & $0,54 \pm 0,53$ & $0,28 \pm 0,19$ & $4,07 \pm 1,68$ \\
\hline P2O5 & $0,16 \pm 0,07$ & $0,25 \pm 0,12$ & $0,12 \pm 0,93$ & $0,16 \pm 0,05$ \\
\hline $\mathrm{H} 2 \mathrm{O}+$ & $0,85 \pm 0,14$ & $4,74 \pm 1,43$ & $3,68 \pm 2,14$ & $3,11 \pm 1,50$ \\
\hline $\mathrm{CO} 2$ & $0,11 \pm 0,04$ & $0,99 \pm 1,01$ & $1,96 \pm 1,78$ & $0,14 \pm 0,01$ \\
\hline $\mathbf{S}$ & $38 \pm 5$ & $63 \pm 26$ & $115 \pm 70$ & $8000 \pm 8000$ \\
\hline $\mathrm{Cl}$ & $68 \pm 25$ & $35 \pm 12$ & $29 \pm 11$ & $30 \pm 6$ \\
\hline $\mathrm{Ba}$ & $270 \pm 55$ & $250 \pm 170$ & $130 \pm 56$ & $800 \pm 190$ \\
\hline $\mathrm{Sr}$ & $460 \pm 31$ & $330 \pm 96$ & $380 \pm 90$ & $110 \pm 64$ \\
\hline $\mathrm{Ni}$ & $61 \pm 36$ & $29 \pm 9$ & $16 \pm 8$ & $13 \pm 13$ \\
\hline v & $280 \pm 23$ & $250 \pm 33$ & $300 \pm 50$ & $130 \pm 99$ \\
\hline $\mathrm{Cu}$ & $106 \pm 17$ & $74 \pm 38$ & $106 \pm 56$ & $56 \pm 66$ \\
\hline $\mathrm{Au}$ & $3,6 \pm 0,7$ & $5,3 \pm 1,3$ & $4,0 \pm 1,6$ & $350 \pm 500$ \\
\hline $\mathrm{Zr}$ & $60 \pm 17$ & $65 \pm 15$ & $60 \pm 21$ & $130 \pm 130$ \\
\hline $\mathrm{Y}$ & $20 \pm 3$ & $19 \pm 4$ & $19 \pm 4$ & $32 \pm 27$ \\
\hline FM & $0,62 \pm 0,06$ & $0,59 \pm 0,05$ & $0,70 \pm 0,03$ & $0,74 \pm 0,10$ \\
\hline OX & $0,99 \pm 0,36$ & $1,17 \pm 0,65$ & $0,84 \pm 1,97$ & $1,93 \pm 15$ \\
\hline $\mathrm{n}$ & 6 & 18 & 8 & 3 \\
\hline
\end{tabular}

\section{RESULTADOS}

Composiciones promedios y desviaciones estándares de las lavas básicas del Grupo Coyol Inferior en las diferentes zonas de alteración se presentan en las Tablas 2 y 3 . Los basaltos no alterados constituyen el valor de fondo geoquímico y su comportamiento es bien uniforme. Desviaciones estándares son bajas para muchos elementos tales como $\mathrm{K}, \mathrm{Ni}, \mathrm{Mg}$ y $\mathrm{Cl}$ (Tabla 2). Una gran parte de la variación detectada es debido a la muestra RAM 15, la cual difiere un poco en composición con respecto a los otros basaltos.

El valor de fondo de las lavas (Tabla 2) es más hidratado y oxidado en comparación con los basaltos recientes y frescos, una matriz de correlación para todas las muestras no alteradas, tanto básicas como ácidas del grupo Coyol Inferior de la región de La Libertad y de sus alrededores demuestra que efectivamente ellas se comportan como muestras sin alteración (Tabla 4). Por ejemplo, $\mathrm{SiO}_{2}$ muestra una correlación positiva fuerte con el $\mathrm{K}_{2} \mathrm{O}, \mathrm{Na}_{2} \mathrm{O}, \mathrm{Ba}, \mathrm{Zr}$, Y OX $\left(=\mathrm{Fe}_{2} \mathrm{O}_{3} / \mathrm{FeO}\right.$; una medida de la oxidación), y con el índice FM (= $\left[\mathrm{FeO}^{*}+\mathrm{MnO}\right] /\left[\mathrm{FeO}^{*}+\mathrm{MnO}+\mathrm{MgO}\right]$, una medida del ínidce de diferenciación definido por Wood, 1978; FeO* es el hierro total) y correlaciones fuertes negativas con $\mathrm{CaO}, \mathrm{MgO}$ y $\mathrm{FeO}^{*}$. Esto significa que las rocas ácidas son más ricas en elementos alcalinos e incompatibles (e.g., Y y Zr) que las básicas.

$\mathrm{El}$ oro presenta una correlación fuertemente positiva con el $\mathrm{Ca} 0, \mathrm{Sr}, \mathrm{Al}_{2} \mathrm{O}_{3}$ y $\mathrm{Mg} 0$ y correlaciones fuertemente negativas con el $\mathrm{SiO}_{2}$ y por lo tanto con los elementos alcalinos, $\mathrm{Ba}, \mathrm{Zr} \mathrm{Y}, \mathrm{OX}$ y FM (Tabla 4).

El cobre muestra correlaciones positivas y negativas con los mismos elementos y relaciones tal como presenta el Au, pero los coeficientes de correlación son mucho menores. En resumen el Au está concentrado en las rocas volcánicas básicas y el $\mathrm{Cu}$ muestra una tendencia similar.

Las correlaciones cambian completamente si únicamente 11 muestras de las lavas básicas no 
Tabla 4

Matriz de correlación de 17 muestras analizadas de rocas volcánicas no alteradas de básicas a ácidas del Grupo Coyol en la región La Libertad y sus alrededores. FeO, FM y OX están explicados en la tabla 2 . Todos los valores han sido normalizados en relación al contenido de Ti $(0,87 \%)$

\begin{tabular}{|c|c|c|c|c|c|c|c|c|c|c|c|c|c|c|c|c|c|c|c|}
\hline & $\mathrm{TiO}_{2}$ & $\mathrm{Al}_{2} \mathrm{O}_{3}$ & $\mathrm{FeO}$ & $\mathrm{MnO}$ & $\mathrm{MgO}$ & $\mathrm{CaO}$ & $\mathrm{Na}_{2} \mathrm{O}$ & $\mathrm{K}_{2} \mathrm{O}$ & $\mathrm{P}_{2} \mathrm{O}_{5}$ & $\mathrm{Ba}$ & $\mathrm{Sr}$ & $\mathrm{Ni}$ & $v$ & $\mathrm{Cu}$ & $\mathrm{Au}$ & $\mathrm{Zr}$ & $\mathrm{Y}$ & $\mathrm{FM}$ & ox \\
\hline $\mathrm{SiO}_{2}$ & $-0,01$ & $-0,68$ & $-0,81$ & $-0,38$ & $-0,89$ & $-0,98$ & 0,96 & 0,97 & $-0,67$ & 0,96 & $-0,75$ & $-0,64$ & $-0,75$ & $-0,57$ & $-0,88$ & 0,93 & 0,86 & 0,92 & 0,86 \\
\hline $\mathrm{TiO}_{2}$ & & $-0,21$ & 0,48 & 0,56 & $-0,18$ & $-0,13$ & 0,25 & 0,12 & 0,07 & 0,29 & $-0,09$ & $-0,37$ & 0,55 & 0,34 & $-0,19$ & 0,30 & 0,47 & 0,15 & $-0,46$ \\
\hline $\mathrm{Al}_{2} \mathrm{O}_{3}$ & & & & 0,63 & 0,41 & 0,75 & $-0,65$ & $-0,82$ & 0,31 & $-0,71$ & 0,80 & 0,18 & 0,57 & 0,33 & 0,81 & $-0,73$ & $-0,76$ & $-0,47$ & $-0,49$ \\
\hline $\mathrm{FeO}$ & & & & 0.50 & 0,72 & 0,75 & $-0,70$ & -0.72 & 0,64 & $-0,62$ & 0,62 & 0,42 & 0,90 & 0,41 & 0,56 & $-0,60$ & $-0,45$ & $-0,73$ & $-0,92$ \\
\hline $\mathrm{MnO}$ & & & & & & 0,37 & $-0,18$ & $-0,44$ & 0,17 & $-0,19$ & 0,53 & $-0,27$ & 0,81 & 0,33 & 0,34 & $-0,20$ & $-0,12$ & $-0,07$ & $-0,58$ \\
\hline $\mathrm{MgO}$ & & & & & & 0,87 & $-0,95$ & $-0,84$ & 0,51 & $-0,90$ & 0,64 & 0,88 & 0.50 & 0,36 & 0,77 & $-0,90$ & $-0,83$ & $-0,99$ & $-0,73$ \\
\hline $\mathrm{CaO}$ & & & & & & & $-0,97$ & $-0,99$ & 0,69 & $-0,97$ & 0,82 & 0,61 & 0,72 & 0,44 & 0,90 & $-0,95$ & $-0,91$ & $-0,89$ & $-0,77$ \\
\hline $\mathrm{Na}_{2} \mathrm{O}$ & & & & & & & & 0,96 & $-0,62$ & 0,98 & $-0,78$ & $-0,74$ & $-0,59$ & $-0,37$ & $-0,89$ & $-0,98$ & 0,94 & 0,96 & 0,72 \\
\hline $\mathrm{K}_{2} \mathrm{O}$ & & & & & & & & & $-0,59$ & 0,96 & $-0,87$ & $-0,58$ & $-0,73$ & $-0,43$ & $-0,92$ & 0,95 & 0,92 & 0,87 & 0,79 \\
\hline $\mathrm{P}_{2} \mathrm{O}_{5}$ & & & & & & & & & & $-0,61$ & 0,36 & 0,22 & 0,55 & 0,33 & 0,45 & $-0,49$ & $-0,50$ & $-0,52$ & $-0,45$ \\
\hline Bă & & & & & & & & & & & $-0,74$ & $-0,71$ & $-0,55$ & $-0,47$ & $-0,92$ & 0,99 & 0,96 & 0,93 & 0,69 \\
\hline $\mathrm{Sr}$ & & & & & & & & & & & & 0,31 & 0,71 & 0,05 & 0,86 & $-0,80$ & $-0,78$ & $-0,68$ & $-0,67$ \\
\hline $\mathrm{Ni}$ & & & & & & & & & & & & & 0,13 & 0,29 & 0,48 & $-0,71$ & $-0,64$ & $-0,84$ & -0.44 \\
\hline v & & & & & & & & & & & & & & 0,44 & 0,60 & $-0,54$ & $-0,41$ & $-0,54$ & $-0,89$ \\
\hline $\mathrm{Cu}$ & & & & & & & & & & & & & & & 0,42 & $-0,42$ & $-0,30$ & $-0,42$ & $-0,61$ \\
\hline $\mathrm{Au}$ & & & & & & & & & & & & & & & & $-0,95$ & $-0,93$ & $-0,83$ & $-0,72$ \\
\hline $\mathrm{Zr}$ & & & & & & & & & & & & & & & & & 0,97 & 0,94 & 0,71 \\
\hline Y & & & & & & & & & & & & & & & & & & 0,86 & 0,54 \\
\hline $\mathrm{FM}$ & & & & & & & & & & & & & & & & & & & 0,77 \\
\hline
\end{tabular}


alteradas son tomadas en cuenta para este tipo de análisis (Tabla 5) . Las previas correlaciones fuertes entre el $\mathrm{Au}$ y otros elementos cambian (las razones de muchos de los elementos con excepción del $\mathrm{Fe} 0 *$ presentan ahora bajos coeficientes o se vuelven débiles $\left(\mathrm{Zr}, \mathrm{Y}, \mathrm{Al}_{2} \mathrm{O}_{3}\right.$ y $\mathrm{Sr}$ ). Por lo tanto el Au presenta una correlación positiva con el $\mathrm{SiO}_{2}$ y negativa con el $\mathrm{Fe} 0 *$ (Fig.3) y el Ni, y correlaciones negativas bajas con el $\mathrm{Zr}$ y el Y. El. Cu incrementa su correlación positiva con el $\mathrm{Ti}_{2}$ y muestra señales de reversión en sus correlaciones con la mayoría de los elementos. Presenta ahora correlaciones positivas con el $\mathrm{Y}$ y los elementos alcalinos y negativas con el Sr y OX. La correlación positiva del $\mathrm{Cu}$ con los elementos alcalinos es debido a que dos muestras contienen valores de $\mathrm{Cu}$ relativamente altos (alrededor de $200 \mathrm{ppm}$ ).

Tabla 5

Coeficientes de correlación para Au y Cu en 11 muestras de lavas básicas no alteradas del Grupo Coyol en la region de La Libertad y sus alrededores

\begin{tabular}{rrr}
\hline & $\mathrm{Au}$ & $\mathrm{Cu}$ \\
\hline $\mathrm{SiO}_{2}$ & 0,65 & $-0,55$ \\
$\mathrm{TiO}_{2}$ & 0,06 & 0,82 \\
$\mathrm{Al}_{2} \mathrm{O}_{3}$ & 0,34 & $-0,04$ \\
$\mathrm{FeO}$ & $-0,86$ & $-0,30$ \\
$\mathrm{MnO}$ & 0,13 & 0,14 \\
$\mathrm{MgO}$ & $-0,37$ & $-0,26$ \\
$\mathrm{CaO}$ & $-0,37$ & $-0,41$ \\
$\mathrm{Na}_{2} \mathrm{O}$ & 0,30 & 0,60 \\
$\mathrm{~K}_{2} \mathrm{O}$ & 0,06 & 0,54 \\
$\mathrm{P}_{2} \mathrm{O}_{5}$ & $-0,26$ & 0,01 \\
$\mathrm{Ba}$ & 0,30 & 0,07 \\
$\mathrm{Sr}$ & 0,55 & $-0,76$ \\
$\mathrm{Ni}$ & $-0,61$ & $-0,03$ \\
$\mathrm{~V}$ & 0,04 & 0,02 \\
$\mathrm{Cu}$ & $-0,16$ & $-0,16$ \\
$\mathrm{Au}$ & & 0,46 \\
$\mathrm{Zr}$ & $-0,40$ & 0,75 \\
$\mathrm{Y}$ & $-0,50$ & 0,75 \\
$\mathrm{FM}$ & $-0,50$ & $-0,66$ \\
$\mathrm{OX}$ & $-0,14$ & \\
& &
\end{tabular}

Las rocas no alteradas del grupo Coyol pertenecen a una región calco-alcalina con afinidad toleítica, tal como señalan Nyström et. al. (1988). El carácter calco-alcalino de los basaltos que fueron considerados como de valores de fondo (background) aparece ilustrado en la Fig.4. El valor bajo de $\mathrm{K}$ en el diagrama indica de que estas rocas podrían haber perdido potasio, lo cual es consistente cuando los comparamos con los basaltos ubicados fuera de la región de La Libertad que presentan valores de $\mathrm{K} 50 \%$ más altos.

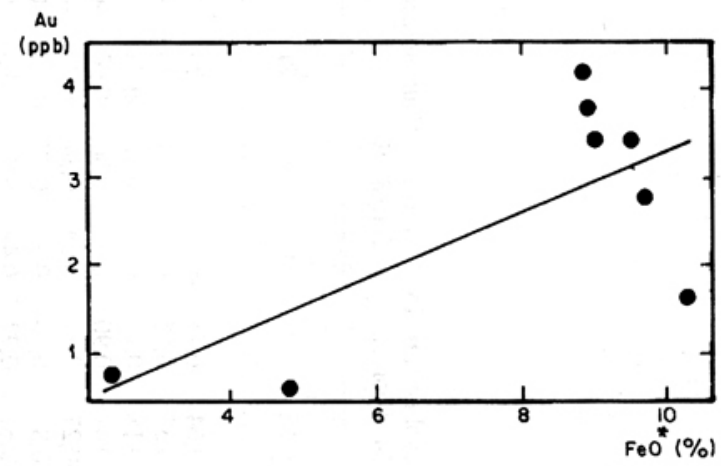

Fig. 3: $\mathrm{FeO}$ versus $\mathrm{Au}$ en todas las muestras de las rocas volcánicas no alteradas del Grupo Coyol analizadas para oro (las muestras pobres en Fe son ácidas, las otras 6 son básicas; Tabla 5). La correlación positiva débil entre al $\mathrm{Au}$ y el $\mathrm{FeO}$ para toda la población (línea de regresión) cambia fundamentalmente a una fuerte correlación negativa si unicamente las lavas básicas son consideradas.

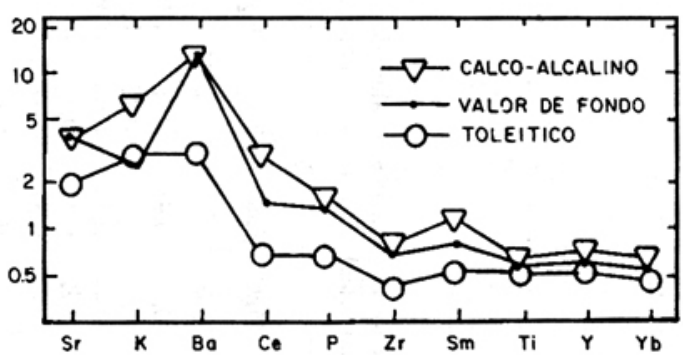

Fig. 4: MORB Patrones geoquímicos normalizados de los basaltos del Coyol Inferior no alterados de la región de La Libertad, usados en esta investigación como valor geoquímico de fondo y análisis representativos de arcos basálticos calcoalcalinos y toleíticos (Pearce, 1982). Los siguientes valores son utilizados para MORB (en ppm. Pearce, 1982): $\mathrm{Sr}=120, \mathrm{~K}_{2} \mathrm{O}$ $=0.15 \mathrm{wt} \%, \mathrm{Ba}=20, \mathrm{Ce}=10, \mathrm{P}_{2} \mathrm{O}_{5}=0.12 \%, \mathrm{Zr}=90, \mathrm{Sm}=$ 3.3, $\mathrm{TiO}_{2}=1.5, \mathrm{Y}=3, \mathrm{Yb}=3.4$.

Los valores de fondo de las lavas revelan una clasificación como "basaltos de alta alúmina” en el límite entre Q-normativo y Ol-normativo. 
Un cálculo de pérdidas y ganancias durante la alteración puede ser hecho de diversas maneras, cada una de ellas involucrando ciertos supuestos y consideraciones (Colman, 1982; Grunsky, 1986). Para poder usar la información analítica de base (Tablas 2 y 3 ) se asume que no han ocurrido cambios de volúmenes y de densidad. Generalmente se asume un volumen constante de las rocas volcánicas durante la alteración hidrotermal debido a que sus texturas y estructuras originales se encuentran bien preservadas (Barth, 1988; Henneberger \& Browne, 1988). La preservación excelente de las texturas volcánicas en las rocas alteradas de la región de La Libertad indican mínimos cambios de volumen.

Medidas acerca de la densidad específica seca de muestras representativas demuestran de que la transferencias de masas y algunos cambios en la densidad han tenido lugar. Muestras de núcleos de perforación alteradas y originalmente permeables de la zona III y IV tienen valores de alrededor 2,35$2,55 \mathrm{gr} / \mathrm{cm}^{3}$ y las lavas masivas tienen valores de entre 2,70 a $2,78 \mathrm{~g} / \mathrm{cm}^{3}$ pareciéndose al valor de fondo de la densidad $\left(2,72 \mathrm{~g} / \mathrm{cm}^{3}\right)$; muestras de la Zona $\mathrm{V}$ y II tienen densidades de $2,25-2,40 \mathrm{~g} / \mathrm{cm}^{3}$. Sin embargo, a pocas muestras se les determinó la densidad por lo que no se tomó en cuenta este método para la corrección.

Un método alternativo para el cálculo de las ganancias y pérdidas, el cual es independiente de la unidad y de los cambios volumétricos durante los procesos de alteración, es la normalización de los análisis por un elemento, asumiendo que dicho elemento permanece constante durante la alteración. El titanio fue escogido para la normalización de los elementos debido a que en muchos estudios se considera como un elemento inmóvil durante la alternación de bajo grado (Liou et al.,1987, y referencias incluidas).

Las composiciones promedios del Ti normalizado para las diferentes zonas de alteración están dadas en las (Tablas 6 y 7). El promedio de cada zona (o sub-grupo) es obtenido al multiplicar los valores de cada muestra por la razón de porcentaje en peso de $\mathrm{TiO}_{2}$ en el valor de fondo (background).

Una comparación de las composiciones promedios antes (Tabla 2-3) y después de la normalización por $\mathrm{TiO}_{2}$ (Tablas 6-7) muestran que dos de estos grupos son más bien similares a los de los núcleos de perforación (excluyendo aureolas) pero difieren más de las muestras de afloramientos, particularmente de aquellas de la Zona II y de la capa caolinítica. Esto es ilustrado por los valores de $\mathrm{SiO}_{2}$. Los métodos asumen un volumen constante durante la alteración (corrección de densidad y el método de celda cation estandar de Barth, 1948, el cual es independiente del cambio de densidad).

Los patrones mineralizados por Ti de las ganancias y pérdidas químicas causadas por la alteración son ilustradas en las Figuras 5 y 6 . Los diagramas sugieren que $\mathrm{H}_{2} \mathrm{O}, \mathrm{CO}_{2}, \mathrm{~K}_{2} \mathrm{O}$ y $\mathrm{S}$ fueron agregados al sistema geotérmico (Zonas II-IV). Los valores de $\mathrm{S}$ incrementan de una manera regular de la zona I hacia las aureolas debajo de la capa caolinítica; la misma tendencia se presenta para el $\mathrm{H}_{2} \mathrm{O}$ en muestras de afloramientos (Tablas 6 y 7). Los valores de $\mathrm{K}_{2} \mathrm{O}$, y la extensión de la oxidación también incrementan. Las aureolas superficiales y sub-superficiales difieren en relación al contenido de $\mathrm{H}_{2} \mathrm{O}(4,6-12 \%$ en la superficie y $1,5-$ $4,4 \%$ en la profundidad).

Tabla 6

Promedios químicos en Tabla 3 (lavas basicas alteradas de afloramientos) normalizados contra el valor de fondo de $\mathrm{TiO}_{2}$ (asumiendo que Ti es inmobil duranbte los procesos de alteracion hidrotermal). Zona $\mathrm{V}$ se refiere a rocas de la capa caolinitica

\begin{tabular}{|c|c|c|c|c|c|}
\hline & $\begin{array}{c}\text { Zona I } \\
\text { Valor fondo }\end{array}$ & Zona II & Zona III & Zona IV & Zona V \\
\hline $\mathrm{SiO}_{2}$ & 50,2 & 46,4 & 55,1 & 58,7 & 47,7 \\
\hline $\mathrm{TiO}_{2}$ & 0,87 & 0,87 & 0,87 & 0,87 & 0,87 \\
\hline $\begin{array}{l}\mathrm{Al}_{2} \mathrm{O}_{3} \\
\mathrm{FeO}\end{array}$ & $\begin{array}{l}18,7 \\
9,30\end{array}$ & $\begin{array}{l}13,8 \\
7,38\end{array}$ & $\begin{array}{l}15,3 \\
7,49\end{array}$ & $\begin{array}{l}19,1 \\
8,06\end{array}$ & $\begin{array}{l}16,6 \\
6,55\end{array}$ \\
\hline $\mathrm{MnO}$ & 0,18 & 0,15 & 0,20 & 0,15 & 0,06 \\
\hline $\mathrm{MgO}$ & 5,86 & 3,57 & 2,68 & 3,62 & 0,43 \\
\hline $\mathrm{CaO}$ & 10,28 & 5,94 & 5,90 & 6,18 & 0,08 \\
\hline $\mathrm{Na}_{2} \mathrm{O}$ & 2,54 & 2,59 & 2,27 & 2,89 & 0,23 \\
\hline $\mathrm{K}_{2} \mathrm{O}$ & 0,38 & 1,04 & 1,63 & 1,77 & 2,28 \\
\hline $\begin{array}{l}\mathrm{P}_{2}^{-} \mathrm{O}_{5} \\
\mathrm{H}_{2} \mathrm{O}^{+}\end{array}$ & $\begin{array}{l}0,16 \\
0,85\end{array}$ & $\begin{array}{l}0,25 \\
1,48\end{array}$ & $\begin{array}{l}0,19 \\
3,69\end{array}$ & $\begin{array}{l}0,18 \\
3,57\end{array}$ & $\begin{array}{l}0,10 \\
5,05\end{array}$ \\
\hline $\begin{array}{l}\mathrm{CO}_{2} \\
\mathrm{~S}\end{array}$ & $0, \frac{11}{38}$ & $0, \frac{12}{38}$ & $\begin{array}{r}0,97 \\
49\end{array}$ & $\begin{array}{r}1,23 \\
81\end{array}$ & $\begin{array}{l}0,11 \\
150\end{array}$ \\
\hline $\mathrm{Cl}$ & 68 & 68 & 48 & 35 & 39 \\
\hline $\mathrm{Ba}$ & 270 & 420 & 560 & 790 & 270 \\
\hline $\mathrm{Sr}$ & 460 & 340 & 160 & 400 & 3 \\
\hline $\mathrm{Ni}$ & 61 & 36 & 15 & 32 & 8 \\
\hline V & 280 & 190 & 190 & 170 & 210 \\
\hline $\mathrm{Cu}$ & 106 & 70 & 107 & 84 & 72 \\
\hline $\mathrm{Au}$ & 3,6 & 0,8 & 1,7 & 2,0 & 13 \\
\hline $\mathrm{Zr}$ & 60 & 120 & 110 & 150 & 83 \\
\hline $\mathrm{Y}$ & 20 & 30 & 29 & 38 & 23 \\
\hline FM & 0,62 & 0,68 & 0,74 & 0,69 & 0,94 \\
\hline OX & 0,99 & 4,2 & 6,4 & 1,71 & 35 \\
\hline $\mathrm{n}$ & 6 & 6 & 5 & 3 & 13 \\
\hline
\end{tabular}


Tabla 7

Promedios químicos de la Tabla 3 (lavas básicas alteradas de las perforaciones) normalizadas contra el valor de fondo $\mathrm{TiO}_{2}$, (asumiendo el Ti como inmobil durante la alteración)

\begin{tabular}{|c|c|c|c|c|}
\hline & \multirow{2}{*}{$\begin{array}{c}\text { Zona I } \\
\text { Valor fondo }\end{array}$} & \multirow{2}{*}{$\begin{array}{c}\text { Zona III } \\
\text { San Antonio }\end{array}$} & \multicolumn{2}{|c|}{ Zona IV } \\
\hline & & & Roca (SJ) & Aureola \\
\hline $\mathrm{SiO}_{2}$ & 50,2 & 52,6 & 49,3 & 85,9 \\
\hline $\mathrm{TiO}_{2}$ & 0,87 & 0,87 & 0,87 & 0,87 \\
\hline $\mathrm{Al}_{2} \mathrm{O}_{3}$ & 18,7 & 17,2 & 18,8 & 23,4 \\
\hline $\mathrm{FeO}$ & 9,30 & 8,98 & 8,85 & 8,47 \\
\hline $\mathrm{MnO}$ & 0,18 & 0,31 & 0,19 & 0,21 \\
\hline $\mathrm{MgO}$ & 5,86 & 6,54 & 3,87 & 2,92 \\
\hline $\mathrm{CaO}$ & 10,28 & 8,86 & 10,48 & 1,01 \\
\hline $\mathrm{Na}_{2} \mathrm{O}$ & 2,54 & 2,84 & 1,97 & 3,02 \\
\hline $\mathrm{K}_{2} \mathrm{O}$ & 0,38 & 0,53 & 0,26 & 5,73 \\
\hline $\begin{array}{l}\mathrm{P}_{2}^{2} \mathrm{O}_{5} \\
\mathrm{H}_{2} \mathrm{O}^{+}\end{array}$ & $\begin{array}{l}0,16 \\
0,85\end{array}$ & $\begin{array}{l}0,26 \\
4,99\end{array}$ & $\begin{array}{l}0,12 \\
3,69\end{array}$ & $\begin{array}{l}0,22 \\
4,32\end{array}$ \\
\hline $\begin{array}{l}\mathrm{CO}_{2} \\
\mathrm{~s}\end{array}$ & $\begin{array}{r}0,11 \\
38\end{array}$ & $\begin{array}{r}1,10 \\
61\end{array}$ & $\begin{array}{l}1,76 \\
105\end{array}$ & $\begin{array}{r}0,19 \\
9000\end{array}$ \\
\hline $\mathrm{Cl}$ & 68 & 34 & 23 & 41 \\
\hline $\mathrm{Ba}$ & 270 & 250 & 120 & 1100 \\
\hline $\mathrm{Sr}$ & 460 & 340 & 380 & 140 \\
\hline $\mathrm{Ni}$ & 61 & 30 & 16 & 19 \\
\hline V & 280 & 260 & 290 & 180 \\
\hline $\mathrm{Cu}$ & 106 & 76 & 98 & 85 \\
\hline $\mathrm{Au}$ & 3,6 & 5,1 & 4,0 & 390 \\
\hline $\mathrm{Zr}$ & 60 & 67 & 56 & 170 \\
\hline $\mathrm{Y}$ & 20 & 19 & 18 & 43 \\
\hline $\mathrm{FM}$ & 0,62 & 0,59 & 0,70 & 0,75 \\
\hline OX & 0,99 & 1,20 & 0,88 & 2,34 \\
\hline $\mathrm{n}$ & 6 & 18 & 8 & 3 \\
\hline
\end{tabular}

Algunos elementos se han perdido parcialmente del sistema (Tablas 6 y 7). Los promedios del Ni y Mg0 son más bajos que los del valor de fondo para todas las zonas (excepto en la zona III de muestras provenientes de núcleos de perforaciones). Ambos elementos muestran una tendencia irregular de valores decrecientes hacia los distritos mineros. $\mathrm{El} \mathrm{Cl}$ es otro elemento lixiviado del sistema, solamente en las muestras de la Zona II presenta valores más altos que el fondo (Tabla 2).

Las Tablas 6 y 7 muestran una variación limitada entre las diferentes zonas para los siguientes elementos en relación al contenido de fondo (no considerando aureolas y capa caolinítica). $\mathrm{Si}_{2}, \mathrm{Al}_{2} \mathrm{O}_{3}, \mathrm{Fe} 0 *, \mathrm{Mn} 0$ (excepto para valores relativamente altos de las muestras de núcleos de la zona III), y $\mathrm{Na}_{2}$ O. La normalizción por Ti demuestra de que la movilidad del $\mathrm{SiO}_{2}$ (Tabla 2) es

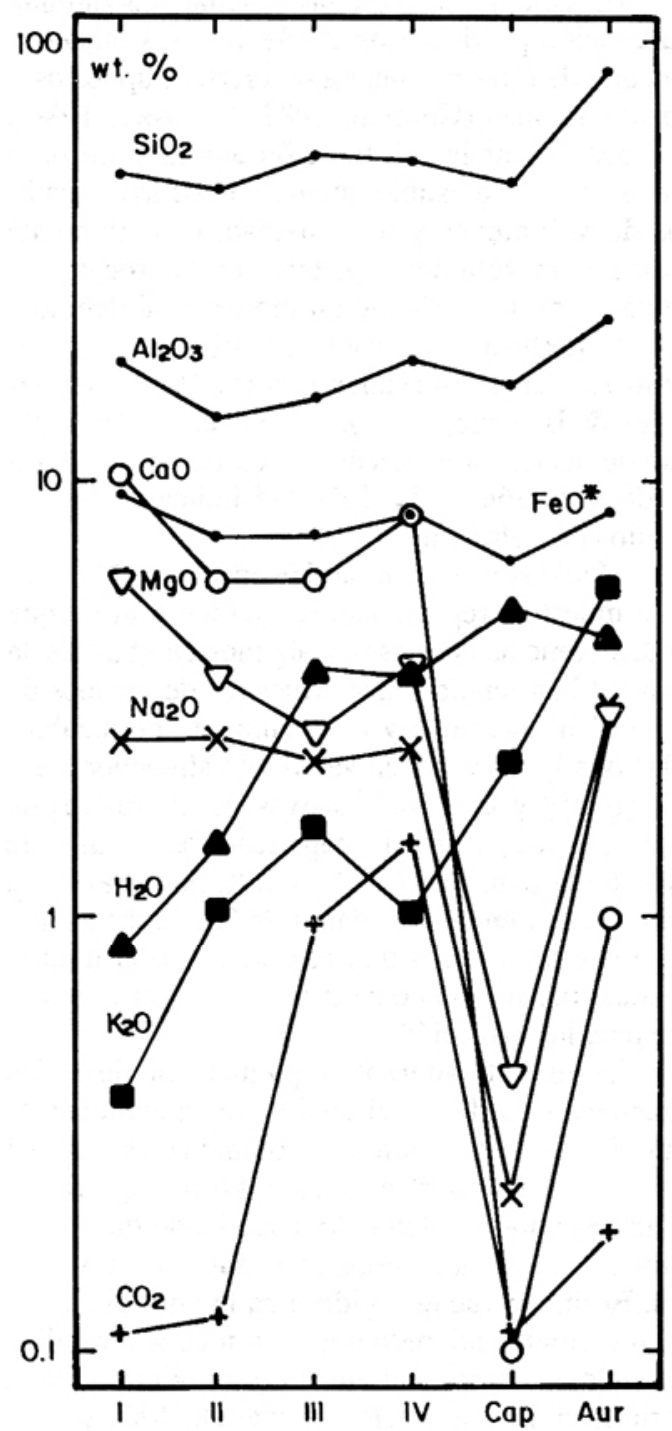

Fig. 5: Curvas de variación normalizadas por Ti para los elementos mayores en las diferentes zonas de alteración en la región de La Libertad. Se ha ploteado el valor promedio para cada zona. Los valores de la Zona I constituyen el valor de fondo. Las muestras de perforaciones de la Zona III no fueron tomados en cuenta por las razones dadas en el texto; la Zona IV indica los promedios de las muestras de afloramientos y perforaciones (aur = aureolas debajo de la capa caolinítica; $\mathrm{FeO}$ es hierro total).

únicamente aparente; por otra parte la normalización indica una deflexión de $\mathrm{Al}_{2} \mathrm{O}_{3}$ en la Zona III. Muestras superficiales son bajas en $\mathrm{Ca} 0$ y $\mathrm{Sr}$ y 


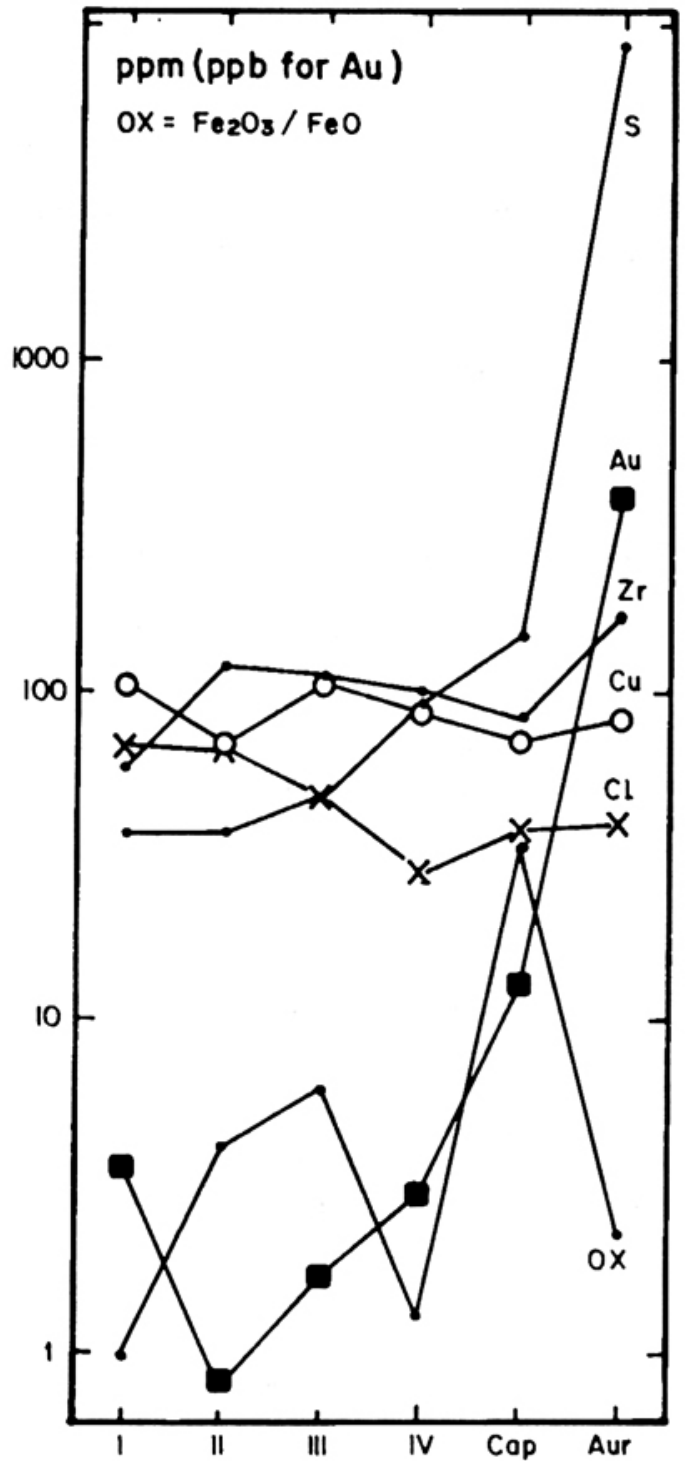

Fig. 6: Curva de variacion normalizada por Ti para elementos trazas y OX que expresan la extensión de la oxidación en las diferentes zonas de alteración de La Libertad (Ver Fig.5 para informacion adicional). La curva para $\mathrm{Y}$ es muy similar a la de Zr y no fue ploteada.

aquellas de aureolas superficiales (y de la capa caolinítica) también son muy bajas en dichos elementos. El comportamiento del Ba es bastante similar al del K (con excepción en capa caolinítica).
El oro se ve disminuido en las rocas superficiales pero los núcleos de las perforaciones tienen valores similares a los de fondo; las aureolas representan enriquecimiento en $\mathrm{Au}$ (y los valores de Au en la capa caolinítica son relativamente altos). $\mathrm{El} \mathrm{Cu}$ por otro lado no varia mucho. Los contenidos son cercanos a los del fondo y a veces más bajos, particularmente en las aureolas. $\mathrm{Zr}$ e $\mathrm{Y}$ son también enriquecidos en la aureolas sub-superficiales y en las muestras superficiales también presentan altos valores.

\section{DISCUSION}

La estimación de lo cambios químicos en las diferentes zonas de alteración de la región de La Libertad está basada en el supuesto de que el valor de fondo (Tabla 2) y el padrón de correlación de la Tabla 5 están soportando la validez general de este supuesto, pero las variaciones no deben de descontarse en sitios de rocas volcánicas comagmáticas. Una de las muestras en el grupo de valor de fondo (RAM 15) difiere químicamente de las otras, sugiriendo que más de un sitio comagmático está presente en la región.

\section{Variaciones químicas debido a la alteración re- gional}

Algunas de las variaciones de los valores de fondo podrían ser parcialmente causadas por la diagénesis de enterramiento o de sepultamiento regional. Durante estos procesos el olivino y el vidrio se reemplazan parcialmente por esmectita y otras fases (Darce, 1989). La alteración parcial del olivino libera $\mathrm{Mg}$ y $\mathrm{Ni}$, los cuales pudieron ser removidos por soluciones. Parte del potasio presente en el vidrio volcánico podría haberse perdido de las rocas. Esto podría explicar el bajo valor del potasio en relación al valor del fondo relativo a las lavas no alteradas del Grupo Coyol en su localidad tipo (cf. Nyström et al., 1988), cuya extensión de alteración es baja y presenta contenidos de $\mathrm{Cl}$ altos. Floyd \& Fuje (1982) reportan que el K y el $\mathrm{Cl}$ fueron extensivamente movilizados durante la alteración de la facies de ceolitas en la parte este de Islandia. Allí, el gradiente térmico fósil (Liou et al.,1987, tabla 3.1) es intermedio entre el gradiente regional y geotérmico de la región de $\mathrm{La}$ Libertad. 


\section{Movilidad de los elementos en el sistema geotérmico fósil}

Es difícil realizar un balance químico de le alteración debido a que el número de muestras analizadas no es grande y ellas están concentradas en una parte limitada de la región. Otra razón es la poca densidad del muestreo. No obstante, la información química reportada en esta investigación permite hacer una estimación cualitativa de las ganancias y pérdidas, mostrando de que cada zona presenta su propia característica geoquímica. La mayoría de las investigaciones que tratan sobre las relaciones entre la alteración y las variaciones químicas se basan en información no corregida para la densidad y cambios de volumen y sin normalización a un elemento inmóvil. Esta aproximación puede ser útil durante actividades de prospección, cuando el propósito es medir únicamente las variaciones de determinados elementos guías. Sin embargo, la normalización contra el Ti, u otro elemento inmóvil o razones entre ellos (Rona et al., 1980, Colman, 1982) próporciona una idea más realistica acerca de las pérdidas y ganancias en un sistema.

Varios estudios han demostrado que el Ti, $\mathrm{Zr}, \mathrm{Y}$, tierras raras y algunos otros elementos se comportan como inmóviles durante la alteración y metamorfismo de muy bajo grado (e.g. Pearce \& Cann, 1973; Nyström, 1984) . La inmóvilidad del $\mathrm{Ti}$ en lavas continentales regionalmente alteradas en la facies de ceolitas ha sido demostrada por Wood et al. (1976) y Robert et al. (1988) y la alteración en las mismas facies en sistemas geotérmicos por Henneberger \& Browne (1988).

El Ti fue escogido para el propósito de normalización en este estudio debido a que al parecer ha sido inmóvil en la región de La Libertad. Inspecciones de secciones delgadas sugieren que el $\mathrm{Ti}$ originalmente presente en la magnetita (en solución sólida o como ilmenita) y en el clinopiroxeno, permanece como producto secundario en o cerca del precursor volcánico que contiene $\mathrm{Ti}$; la ilmenita es resistente a la alteración excepto en la capa caolinítica y en las aureolas. Por ejemplo, minerales seudomorfos según piroxeno, conteniendo titanita son comunes en la zonas II a IV.

El $\mathrm{Zr}$ no fue usado para la normalización, debido a que Widenfalk \& Altamirano (1985) reportaron movilidad del $\mathrm{Zr}$ en las aureolas de vetas de cuarzo en El Limón (Fig.1), un depósito parecido a La Libertad. Ellos encontraron que $\mathrm{SiO}_{2}$,
$\mathrm{K}_{2} \mathrm{O}, \mathrm{Zr}$ (y Hg) han sido adicionados a las aureolas en dos sistemas de vetas estudiadas, mientras que otros elementos fueron sustraídos del sistema.

Como se puede ver en la Tabla 7 los mismos elementos están enriquecidos en las aureolas de La Libertad. Los relativamente altos valores de $\mathrm{Zr}$ e $\mathrm{Y}$ en la parte superior y externa del sistema geotérmico fósil son probablemente más aparentes que reales y están reflejando variaciones primarias en las proporciones de $\mathrm{Ti}, \mathrm{Zr}$ e $\mathrm{Y}$. Tales variaciones son mostradas por los valores de fondo de los basaltos no alterados.

Otro elemento considerado como relativamente inmóvil en muchos casos es el Al. Sin embargo no fué escogido para la normalización debido a que los minerales que contienen $\mathrm{Al}$ (por ejemplo:caolinita y adularia) son desarrollados en rocas lixiviadas ácidas cercanas a la mineralización. El Al puede ser transportado en soluciones alcalinas en los campos geotérmicos (Henneberger \& Browne, 1988). Las asociaciones pertenecientes a la facies de laumontita en las zonas III y IV indican condiciones alcalinas.

\section{Relaciones entre mineralogía y quimismo}

La característica geoquímica de cada zona es una expresión de los cambios en la mineralogía que han tenido lugar enel distrito minero. Ambos fenómenos reflejan las diversas extensiones y escalas de movilidad de los diversos elementos y la variación en condiciones físico-químicos durante la interacción flúido-roca en un sistema abierto. El K adicional al sistema geotérmico (Zonas II-IV) probablemente se derivó de la descomposición del vidrio volcánico en la zona I lo cual tuvo su expresión en la formación de heulandita, mordenita y esmectita. La formación de ceolitas sugiere que las soluciones fueron neutrales a ligeramente alcalinas (Seki et al., 1983; Liou et al. 1985; Henneberger \& Browne, 1988), consistente con la ausencia de caolinita. La falta de calcita se debe probablemente a la baja actividad de $\mathrm{Ca}$ en las soluciones.

En las zonas III la laumontita toma el lugar de las ceolitas que contienen $\mathrm{K}$ y está limitado a la clorita expandible (o esmectita). La formación de laumontita está controlada por el incremento en la temperatura, un relativamente moderado alto $\mathrm{pH}$ y alta actividad de $\mathrm{Ca}$. La plagioclasa cálcica, que está esencialmente no alterada (o parcialmente reemplazada por heulandita) en la zona I y II, requicbra en la zona III. El Ca liberado se incorpora 
en la laumontita y calcita; algo de Ca también proviene de la alteración de los clinopiroxenos.

Condiciones menos alcalinas y más reductoras se reflejan en bajos valores de OX y prevalecen más que todo en la zona IV. Esto es soportado por la presencia de illita y clorita y la ocurrencia local de pirita y caolinítica (no se podrá excluir completamente la idea de que la caolinita es de un desarrollo posterior). El K se presenta en la illita y la clorita expandible y el $\mathrm{Ca}$ en los mismos minerales como en la zona III (relictos de plagioclasaclinopiroxeno en la parte masiva de la lavas a profundidad, con una adición local de epidota y wairakita en donde las temperaturas fueron altas (Boles, 1981, Henley \& Ellis, 1983). La sustracción del $\mathrm{K}$ y del $\mathrm{Ba}$ en las muestras de la perforaciones de la zona IV está probablemente relacionada al desarrollo abundante de feldespato en las cercanías de las aureolas (Tabla 7).

La capa supergénica es de un desarrollo posterior (Darce, 1989), sin embargo, todavía no se puede asegurar de que las aureolas de sub-superficie sean más jóvenes y de formación coevales en las zonas de alteración III y IV que contienen ceolitas consistente con la abundancia del feldespato-K en las aureolas y la sustracción relativa del $\mathrm{K}$ en la zona IV. Ninguna calcita y laumontita originalmente presente pudo escaparse a la reacción de las soluciones ácidas tardías, explicando de esta manera la gran pérdida en las capas caolinitas y en las aureolas. La lixiviación es también responsable por el bajo contenido de $\mathrm{Na}$ en la capa caolinítica; en las aureolas sub-superficiales el $\mathrm{Na}$ permanece en la albita.

Pequeñas incrustaciones de hidróxido de Mn se encuentran en las fracturas de las rocas de la capa caolinítica cercana a las vetas de cuarzo. El promedio del contenido de Mn en la capa caolinítica se baja ya que las muestras ricas en Mn no fueron analizadas (por ejemplo los valores de las aureolas; Tabla 7). La razón para el relativamente alto contenido de $\mathrm{Mn}$ en las muestras de las perforaciones de la zona III no es todavía conocida. Estas muestras son anómalas en varios aspectos (por ejemplo: algunas veces, sus contenidós de Au son mayores que los valores de fondo). La proximidad a una veta de cuarzo potencialmente aurífera, indicada por el desarrollo de una mineralogía típica de aureola en la parte inferior de la perforación de San Antonio (Darce, 1989), sugiere de que la geoquímica de estos núcleos a profundidad no son enteramente representativos de la zona III. Lamenta- blemente no fué puesto ningún énfasis en la anomalía de ésta perforación.

\section{Las rocas volcánicas como fuente de oro en La Libertad}

La tendencia del Au de ser más abundante en las rocas básicas que en las ácidas (ambas no alteradas) es demostrada en las correlaciones de la Tabla 4 y son de una validez general (Boyle, 1979). Zentilli et al. (1985) investigaron una secuencia Miocénica de basaltos toleíticos y de andesitas basálticas de Islandia, alteradas en las facies de ceolitas, usando muestras de una perforación profunda (The Iceland Research Drilling Project) y de los niveles estratigráficos superiores. Los autores concluyeron que el oro fué movilizado dentro de la alteración. En muestras de perforación poco alteradas, el oro presenta una correlación positiva con elementos incompatibles tales como $\mathrm{Y}, \mathrm{Zr}$ y Rb. Esto indica de que el oro es enriquecido en los líquidos residuales y puede finalmente ser atrapado como minerales accesorios posteriores y como vidrio en las mesostasa, además de los sulfuros.

Sin embargo, en las muestras de afloramientos de la parte superior de las secuencias en donde las lavas son más del tipo de cuarzo-normativos, las correlaciones del Au con el $\mathrm{Zr}$ y el $\mathrm{Y}$ son negativas. Zentilli et al. (1985) interpretaron estas correlaciones como "primarias".

Las rocas básicas analizadas de la región de La Libertad están alteradas en un grado similar por representar un rango más limitado con relación al $\mathrm{SiO}_{2}$. Las correlaciones de la tabla 5 se asemejan a aquellas muestras de afloramientos usadas por Zentilli (1985). Las sugerencias de estos autores y del presente estudio de que el oro está concentrado en las mezclas de silicatos residuales (vidrio) está en desacuerdo con investigaciones anteriores (Gottfriend et al., 1972, Tilling et al., 1973). Una concentración de oro en el vidrio de las mesostasa es consistente con dos circunstancias: está positivamente correlacionada con $\mathrm{Si}_{2}$ y el $\mathrm{Na}_{2} 0$, y negativamente correlacionada con $\mathrm{Fe} 0 *$, $\mathrm{Ni}, \mathrm{Mg} 0$ y $\mathrm{Ca} 0$ (Tabla 5). Aún más, la sustracción fuerte del oro que ha tenido lugar en la zona II, podría estar relacionada con la más importante reacción de alteración: el reemplazamiento del vidrio y del olivino por la esmectita. Las correlaciones negativas anteriormente descritas argumentan en contra de que el olivino sea una importante fuente de Au. 
De acuerdo a Keays \& Scott (1976) soluciones ricas en $\mathrm{Cu}, \mathrm{Ni}, \mathrm{Mg}$ ayudan a la movilización del oro. La descomposición del olivino en la zona II podría suplir cantidades adecuadas de $\mathrm{Ni}$ y $\mathrm{Mg}$.

Las facies de alteración de ceolitas, como en la zona II y III, están caracterizadas por condiciones oxidantes de soluciones termales neutras-alcalinas, las cuales son favorables para la lixiviación y transporte del Au así como de complejos que contienen S (Oyarzun 1984). La lixiviación es demostrada por la existencia de relictos de lavas masivas no alteradas con contenidos de oro similares a los valores de fondo. Información de isótopos de plomo de Sundblad et al. (1989) soportan la lixiviación de $\mathrm{Pb}$ de la roca volcánica circundante.

Estudios de depósitos epitermales indican que la naturaleza de las soluciones hidrotermales es predominantemente meteórica (Field \& Fifarek, 1985). El Au lixiviado fué transportado en solución o quizás como coloide. Complejos con S, As, $\mathrm{Sb}$ y $\mathrm{Cl}$ (como $\mathrm{HS}^{-}, \mathrm{S}^{2-}, \mathrm{AsS}^{2-}$, etc.) pueden formar complejos estables con el Au y producen un transporte efectivo en las soluciones térmicas (Boyle, 1979). De acuerdo a la hipótesis de Frondel (1938) de que el oro coloidal es estabilizado por la presencia de un segundo coloide protectivo (sílica), realmente nunca ha sido aprobada o desaprobada (Park \& MacDiarmid, 1975). La sustracción de Au en la mayoría de las muestras superficiales relativas a los valores del fondo y los valores de las muestras de perforaciones es una indicación de meteorización. La esmectita puede formarse sola durante la meteorización, no así las asociaciones mineralógicas secundarias de las muestras de los afloramientos de las zonas II a IV. Estudios petrográficas de las vetillas de esmectita, ceolitas y cuarzo indican de que la alteración se formó en el mismo tiempo. Alguna pérdida de $\mathrm{Au}$ durante la sub-reciente meteorización es posible, pero ella sola no puede ser responsable para el origen de las vetas auríferas de La Libertad. La presencia de fuentes calientes, fracturamiento y condiciones hidrólogicas generales son pre-requisitos para el origen de un depósito epitermal como el de La Libertad, más importantes aún que un relativamente alto contenido de $\mathrm{Au}$ en la roca circundante (Henley, 1985). Los patrones mineralógicos en la región de La Libertad requieren de la presencia de una fuente de calor bajo el campo geotérmico fósil, es decir del presente distrito minero. Darce
(1989) postuló la existencia de una intrusión de alto nivel. Los cambios mineralógicos y químicos en las rocas fracturadas son consistentes con soluciones calientes circulantes y gradientes físicoquímicos locales. Las fracturas y las rocas permeables fueron sellados por la deposición del sílice y otras fases secundarias recurrentes como fracturamiento hidráulica en un área tectónicamente activa que causó la reapertura de nuevos canales.

Está más allá del objetivo de esta investigación discutir los factores que controlan el transporte y depositación de Au. Los principales factores de control pueden ser el decrecimiento de los valores de $\mathrm{pH}$ y de salinidad; ebullición, dilución y oxidación de acuerdo con Boyle (1979), Berger \& Beth (1985) y Crerar et al. (1985). Los patrones mineralógicos de la alteración están expresados en las aureolas superficiales y se reflejan en los estudios de inclusiones flluidas de las vetas de cuarzo (Halenius, comunicación verbal, 1986; Morales et al., 1993), y son análogos de los sistemas geotérmicos activos (Henley, 1985).

\section{Balance del oro}

Un estimado de la cantidad de Au lixiviado durante la alteración geotérmica de las rocas circundantes da una cantidad similar a la que existe en el depósito de La Libertad. Asumiendo de que las rocas no alteradas contienen 3,6 ppb Au (valor de fondo) y que $2 / 3$ partes del Au son lixiviados, un volumen de roca de $980 \mathrm{~km}^{3}$ es necesario para producir 6,5 ton Au. que hay en el depósito (1,3 x $10^{6}$ toneladas mineralizadas con leyes de $5 \mathrm{gr} \mathrm{Au}-$ /ton). Dos terceras partes de esta cantidad está en las zonas II y III, no tomando en cuenta los valores anómalos de Au de la perforación en San Antonio. La extrapolación de los límites de las zonas en la figura $2 \mathrm{~A}$ da un área del orden de los 920 $\mathrm{km}^{2}$ para las zonas II y III. Una lixiviación actuando hasta una profundidad de $1060 \mathrm{~m}$ podría suplir la cantidad de Au necesario para formar el depósito de La Libertad.

Para realizar un real balance del oro, hace falta conocer varios factores de importancia. Son muy difíciles de estimar factores tales como: la erosión del depósito, contenido de Au en la Formación Matagalpa, profundidad de lixiviación real, la extensión de alteración del Grupo Coyol, los espesores de la zona II y III. 


\section{Comportamiento del cobre}

Los contenidos de $\mathrm{Cu}$ tienden a ser más altos en las rocas básicas que en la ácidas (Gill, 1978) y en las series toleíticos más que en las calco-alcalinos.

Este comportamiento se refleja muy bien en las rocas volcánicas no alteradas Terciarias y recientes de Nicaragua (Nyström et al., 1988). Una parte del $\mathrm{Cu}$ (igual al $\mathrm{Au}$ ) parece estar concentrado en el vidrio (J.O. Nyström, información inédita). Esta interpretación es consistente con las correlaciones entre el $\mathrm{Cu}$ y de algunos otros elementos de las lavas básicas no alteradas (Tabla 5): positiva con los elementos alcalinos, $\mathrm{Y}$ y $\mathrm{Zr}$ y negativa con $\mathrm{Sr}, \mathrm{Ca} 0, \mathrm{Fe} 0 *$ y $\mathrm{Mg} 0$. Sin embargo, la correlación negativa con el $\mathrm{Si}_{2}$ y la positiva con el $\mathrm{TiO}_{2}$ son inconsistentes. Enriquecimiento de $\mathrm{Cu}$ en la fase de vapor tardía fue indicado por Eillenberg \& Carr (1981).

Las lavas recientes y terciarias no alteradas contienen pequeñas cantidades de calcopirita, calcosita y bornita. En muestras de lavas recientes estos minerales están dentro de la magnetita oxidada, demostrando de esta manera que los sulfuros son productos secundarios, quizás debido a una oxidación posterior al enfriamiento.

El cobre y el Au reaccionaron diferentemente a la alteración. El oro fue lixiviado y removido, mientras que el $\mathrm{Cu}$ permaneció en las rocas como sulfuro. La mayoría del S necesario para esta reacción podría haber sido suplido por la roca misma. El cobre se perdió parcialmente en las aureolas, en donde la alteración es fuerte. Durante la alteración supergénica de la capa caolinítica el Cu no sufrió grandes variaciones.

\section{Depositos epitermales y sistemas geotérmicos}

Los depósitos epitermales de la faja volcánica Circum-Pacífica ocurren en el mismo marco geológico de los campos geotérmicos activos (Henley, 1985). Regímenes tensionales tales como márgenes de caldera y grabenes proveen condiciones favorables para la concentración de metales en sistemas térmicos. (Levy, 1970; Sillitoe, 1977; Lipman et al., 1984; Oyarzún, 1984; Hayba et al., 1985, Henley, 1985). Hedenquist \& Henley (1985) y Henley (1985) reportan que las mineralizaciones económicas de oro se han precipitado en campos geotérmicos activos. La presente investigación de un sistema geotérmico fósil demuestra que la extensiva lixiviación de la roca volcánica circundante puede formar depósitos epitermales auríferos.

\section{AGRADECIMIENTOS}

Agradezco especialmente a B.Levi y a J.O. Nyström por su guía en esta investigación. W. Vivallo, $\mathrm{K}$. Bostrom y V. Morogam criticaron y ayudaron a mejorar el manuscrito. J. Arnstrom y S. Jevall dibujaron las figuras. B. Bostrom me ayudó en procedimientos analíticos de laboratorio y $\mathrm{K}$. Ubieta realizó pruebas de densidad en algunas muestras. E. Emes mecanografió el texto de forma muy eficiente y J.C. Tablada ayudó en la confección de tablas y figuras. Este estudio fue auspiciado por INMINE y SAREC.

\section{REFERENCIAS}

Barth, T.F.W., 1948: Oxygen in rocks: a basis for petrographic calculations. - J. Geol., 56: 5060.

Berger, B.R. \& Bethke, P.M., 1985 (eds.): Geology and geochemistry of epithermal systems. - Rev. Econ. Geol., 2, 298 pp.

Boles, J.R.,1981: Zeolites in low grade metamorphic rocks. - In: Mumpton, F.A. (ed.): Mineralogy and geology of natural zeolites. Rev. Mineral., 4: 103-135.

Boyle, R.W.,1979: The geochemistry of gold and its deposits. - Geol.Surv.Canada Bull., 280, $584 \mathrm{pp}$.

Burman, J.O., Boström, B.\& Boström, K.,1977: Geochemical analyses by plasma spectroscopy. - Geol. Föreningens Stockholm Förhandlingar, 99: 102-110.

Burman, J. O., Porter, C.\& Boström, K., 1978: Metaborate digestion procedure for inductively coupled plasma-optical emission spectrometry. - Analyt. Chem., 50: 679-680.

Colman, S. M., 1982: Chemical weathering of basalts and andesites: evidence from weathering rinds. - U. S. Geol. Surv. Prof. Paper,. $1246,51 \mathrm{pp}$. 
Crerar, D., Wood, S., Brantley, S.\& Bocarsly, A., 1985: Chemical controls on solubility of ore forming minerals in hydrothermal solutions. - Canad. Mineralog., 23: 333-352.

Darce, M., 1987: Geología del distrito minero La Libertad, Nicaragua. - Rev. Geol. América Central, 7: 65-82.

Darce, M., Levi, B. \& Nyström, J.O., 1991: Chemical changes during alteration of volcanic rocks and gold ore formation, La Libertad, Nicaragua. - J. South Amer, Earths Sci., 4(1-2): 87-97.

Darce, M., 1990: Mineralogic alteration patterns in volcanic rocks of La Libertad gold mining district and its surroundings, Nicaragua. - Econ. Geol. 85: 1059-1071.

Darce, M., Levi, B., Nyström, J.O. \& Troëng, B., 1989: Alteration pattern in volcanic rocks within an east west traverse through central Nicaragua: J. South Amer. Earth Sci., 2: 155-161.

Dengo, G., 1985: Mid America: Tectonic setting for the Pacific margin from southern Mexico to northwestern Colombia. - In :Naim, A.E.M., Stehli, F.G. \& Uyeda, S. (eds.): The ocean basins and margins, v.7A, Plenum Press, New York, London: 123-180.

Dengo, G. \& Levy, E., 1970: Anotaciones al mapa metalogenético de América Central. - Publ. geol. Instituto Centroamericano de Investigacion y Teccnologia Industrial, Guatemala, 3: 1-15.

Dostal, J., \& Dupuy, C., 1987: Gold in late Proterozoic andesites from northwesterm Africa: - Econ. Geol., 82: 762-766.

Eilemberg, S. \& Carr, M.J., 1981: Copper contents of lavas form active volcanoes in El Salvador and adjacent regions in Central American. - Econ.Geol., 76: 2246-2248.

Field, C.,W. \& Fifarek. R.H., 1985: Light stable isotope systematics in the epithermal envi- ronment. - In: Berger, B.R. \& Bethke, P.M. (eds.): Geology and geochemistry of epithermal systems. - Econ.Geol., 2: 99-128.

Floyd, P.A. \& Fuge, R., 1982: Primary and secondary alkali and halogen element distribution in Iceland Research Drilling Project basalts from easterm Iceland. - J. Geophys. Res., 87B: 6477-6488.

Frondel, C., 1938: Stability of colloidal gold under hydrothermal conditions. - Econ.Geol., 33: 1-20.

Gill, J.B., 1978: Role of trace element partition coefficients in models of andesite genesis. Geochim Cosmochim Acta, 42: 709-724

Gottfried, P., Rowe, J.J. \& Tilling, R.I., 1972: Distribution of gold in igneous rocks. - U.S. Geol. Surv. Prof.Paper, 727: 42 pp.

Govindaraju, K., \& Mevelle, G., 1987: Fully automated dissolution and separation methods for inductively coupled plasma atomic emission spectrometry rock analysis. Aplication to the determination of rare earth elements. - J. Analyt. Atomic Spectrometry, 2: 615-621.

Grunsky, E.C., 1986: Recognition of alteration in volcanic rocks using statistical analysis of lithogeochemical data. - In: Wood, J. \& Wallace, H. (eds.): Volcanology and mineral deposits. - Ontario Geol. Surv. Misc. Paper, 129: $125-173$.

Hålenius. U., 1983: A mineralogical investigation of the gold bearing vein type deposits at $\mathrm{Li}$ bertad, El Limon and Rincón de García, Nicaragua. - INMINE/SAREC/SGAB unpub.rept., Swedish Geological Company, Luleå, $15 \mathrm{pp}$.

Hayba, D.O., Bethke, P.M., Heald, P. \& Foley, N.K., 1985: Geologic, mineralogic and geochemical characteristics of volcanic hosted epithermal precious metal deposits. - In: Berger, B.R. \& Bethke, P.M. (eds.): Geology and geochemistry of epithermal system. - Econ. Geol.. 2: 129167. 
Hedenquist, J.W. \& Henley, R. W., 1985: Hydrothermal eruption in the Waiotapu geothermal system, New Zeland; their origen, associated breccias, and relation to precious metal mineralization. - Econ. Geol., 80: 16401668.

Hellman, P.L., Smith, R.E. \& Henderson, P.,1979: The mobility of the rare earth element: evidence and implication from selected terrains affected by burial metamorphism. - Contrib. Mineral. Petrol.,.71: 23-44.

Henley, R., W., 1985: The geothermal framework of epithermal deposit. - In: Berger, B. R. \& Bethke, P. M. (eds.): Geology and geochemistry of epithermal systems. - Econ. Geol., 2: $1-24$

Henley, R.W. \& Ellis, A. J., 1983: Geothermal system ancient and modern: a geochemical review. - Earth Sci. Rev., 19: 1-50.

Henneberger, R.C. \& Browne, P.R.L., 1988: Hydrothermal alteration and evolution of the Ohakuri hydrothermal system, Taupo Volcanic Zone, New Zealand. - J. VolcanolGeotherm. Res., 34: 211-231.

Keays, R.R. \& Scott, R.B., 1976: Precious metals in ocean ridge basalts: implications for basalts as source rocks for gold mineralization. - Econ.Geol., 71: 705-720.

Levy, E. 1970, La metalogénesis en América Central. - Publ. Geol. Instiuto Centroamericano de Investigación Industrial, Guatemala, No.3: 17-76.

Lindgren, W., 1928, Mineral deposits. - McGraw Hill (New York and London), 3rd ed., 1049 pp.

Liou, J.G, Seki, Y., Guillemette, R, N. \& Sakai, H., 1985: Compositions and parageneses of secondary minerals in the Onikobe geothermal system, Japan. - Chem. Geol., 49: 1-20.

Liou, J. G., Maruyama, S. \& Cho, M., 1987: Very low grade metamorphism of volcanic and volcaniclastic rock mineral assemblage and mineral facies. - In: Frey, M. (ed): Low tem- perature metamorphism. - Blackie (Glasgow and London): 59-113.

Lipman, P.W., Self, S.,S. \& Heiken, G., 1984: Introduction to Calderas. - Special Issue J. Geophys. Res, 89B: 8219-8221.

Morales, A., Vivallo, W. \& Darce, M., 1993: Fluid inclusion studies of gold bearing quartz veins in the La Libertad district, Nicaragua. - Rev. Geol. América Central, 16: 23-38.

Nyström, J.O., 1984: Rare earth element mobility in vesicular lava during low grade metamorphism. - Contrib. Mineral.Petrol., 88: 328-331.

Nyström,J.O., Levy, B., Troëng, B., Ehrenborg, J. \& Carranza, G., 1988: Geochemistry of volcanic rocks in a traverse through Nicaragua. - Rev. Geol.América Central, 8: 77-109.

Oyarzún, J., 1984: Geoquímica y metalogénesis endógena de oro. - Rev. Geol.Chile, 24: 310.

Park, C.F. \& MacDiarmid. R.A., 1975: Ore deposits. - Freeman and Company (San Francisco), 3rd ed., 529 pp.

Pearce, J. A., 1982: Chemical and isotope characteristics of destructive margin magmas. - In: Thorpe, R. S.(ed.): Andesites: orogenic andesites and related rocks.- John Wiley \& Sons (New York): 525-548.

Pearce, J.A. \& Cann, J.R., 1973: Tectonic setting of volcanic rocks determined using trace element analyses. - Earth Planet Sci. Lett., 19: 290-300.

Robert, C., Goffé, B. \& Saliot, P., 1988: Zeolitization of a basaltic flow in a continental environment: an example of mass transfer under thermal control. - Bull. Mineral., 111: 207 223.

Rona, P.A., Boström, K. \& Epstein, S.,1980: Hydrothermal quartz vug from the Mid Atlantic Ridge. - Geology, 8: 569-572. 
Seki, Y., Liou, J.G., Guillemette, R., Sakai, H., Oki, Y., Hirano, T. \& Onuki, H., 1983: Investigation of geothermal systems in Japan, I: Onikobe geothermal area. - Hydrosci. Geotech.Lab., Saitama Univ., Mem., 3, 206 pp

Sillitoe, R.H.,1977: Metallic mineralization affiliated to subaerial volcanism: a review. Geol. Sc.London, Spec., Publ., 7: 99-116.

Sundblad, K., Cumming, G. L. \& Krstic, D., 1989: Lead isotope evidence for the formation of epithermal gold quarz veins in the Chortis Block, Nicaragua. - Econ.Geol. 86: 944-959.

Tilling, R.I., Gottfried, D. \& Rowe, J.J., 1973: Gold abundance in igneous rocks: bearing on gold mineralitization. - Econ. Geol., 68: 168-186.

White, D. E., 1955: Thermal springs and epithermal ore deposits. - Econ. Geol., 50 th Anniv. vol.:99-154.
White, D. E., 1981: Active geothermal systems and hydrothermal ore deposits. - Econ. Geol., 75 th Anniv. vol.: 392-423.

Widenfalk, L. \& Altamirano, G., 1985: Distribution of main and trace elements in the wallrocks at the Panteon vein, S: A Pancha, El Limón, Nicaragua. - INMINE/SAREC/SGAB unpub. rept., Swedish Geological Company, Luleå, $56 \mathrm{pp}$.

Wood, D.A., 1978: Major and trace element variations in the Tertiary lavas of eastern Iceland and their significance with respect to the Iceland geochemical anomaly. - Jour. Petrol., 19: 393-436.

Wood, D. A., Gibson, I L. \& Thompson, R.N.,1976: Elemental mobility during zeolite facies metamorphism of the Tertiary basalts of eastern Iceland. - Contrib. Mineral. Petrol. 55: 241-254.

Zentilli, M., Brooks, R.R., Helgason, J., Ryan, D. E. \& Zhang, H., 1985: The distribution of gold in volcanic rocks of eastern Iceland. Chem. Geol., 48: 17-28. 